\title{
Über das Zusammenwirken von menschlicher und künstlicher Intelligenz aus ethischer Sicht
}

\author{
Andreas Kruse
}

\section{Zusammenfassung}

Der nachfolgende Beitrag konzentriert sich auf das Zusammenwirken von menschlicher und künstlicher Intelligenz im Alter. Er untersucht dieses Zusammenwirken im Kontext von Selbstgestaltung, Weltgestaltung und Verletzlichkeit. Selbst- und Weltgestaltung werden als zwei grundlegende Motive des hohen Alters verstanden: es wird diskutiert, in welcher Hinsicht künstliche Intelligenz in ihrem Zusammenwirken mit menschlicher Intelligenz zur Verwirklichung dieser beiden Motive beizutragen vermag. Weiterhin werden die kompensatorischen Funktionen dieses Zusammenwirkens im Kontext der Verletzlichkeit diskutiert, wobei Verletzlichkeit als bedeutender Aspekt der Conditio humana interpretiert wird, auf den Menschen antworten müssen. Die Nennung von Prinzipien der ethischen Betrachtung digitaler Technologien soll als allgemeiner ethischer Orientierungsrahmen des Zusammenwirkens

Das Manuskript basiert auf einem am 7. Oktober 2019 gehaltenen Vortrag in einer von der Braunschweigischen Wissenschaftlichen Gesellschaft, der Ev.-luth. Kirchengemeinde St. Katharinen Braunschweig und der Evangelischen Studierendengemeinde Braunschweig organisierten Veranstaltung „Die Welt, in der wir leben - wollen“. Dort umrahmte der Verfasser seinen Vortrag mit dem Präludium und der Fuge in cis-Moll von Johann Sebastian Bach (BWV 849).

\footnotetext{
A. Kruse $(\bowtie)$

Institut für Gerontologie der Ruprecht-Karls-Universität Heidelberg, Heidelberg, Deutschland

E-Mail: andreas.kruse@gero.uni-heidelberg.de
}

(C) The Author(s) 2021 
von menschlicher und künstlicher Intelligenz dienen; Dilemmata der Nutzung digitaler Technologien werden aufgezeigt und diskutiert. Der fachlich und ethisch verantwortliche Umgang mit künstlicher Intelligenz wird als eine Chance für ein gutes Leben im Alter gewertet.

\section{Schlüsselwörter}

Selbstgestaltung · Weltgestaltung · Verletzlichkeit $\cdot$ Kompetenz $\cdot$ Potenziale $\cdot$ Prinzipien ethischer Betrachtung $\cdot$ Ethische Dilemmata $\cdot$ Gesellschaftliche Verantwortung

\section{Einführung: Zur Würde des Menschen}

Die ethischen Fragen der Künstlichen Intelligenz stehen im Vordergrund dieses Beitrags. Dabei soll der ethische Diskurs kontextualisiert werden: durch den Vortrag des Präludiums und der Fuge in cis-Moll, Wohltemperiertes Klavier, Band 1, von Johann Sebastian Bach (1685-1750). Erscheint eine derartige Rahmung angemessen für das mir aufgegebene Thema? In meinem Redebeitrag konnte ich Präludium und Fuge vortragen; in meinem schriftlichen Beitrag kann ich diese beiden Werke nur mit Worten ausdeuten. Aber auch diese Deutung wird zeigen: tiefer gehende ethische Fragen lassen sich sehr wohl mit den beiden genannten Werken von Johann Sebastian Bach kontextualisieren, weil uns diese Werke - bei näherem Hinschauen - zu uns selbst führen, mithin eine Introversion mit Introspektion anstoßen, die für die ethische Reflexion bedeutsam ist. Denn die ethische Reflexion berührt in zentraler Weise die sittlich-moralischen Grundlagen unseres Entscheidens und Handelns; und diese Grundlagen können wir nur in dem Maße erfassen, in dem wir in der Lage sind, in uns hineinzuhören. Dieses Hineinhören führt uns zu kognitiven Erkenntnissen, aber auch zu emotionalen und motivationalen Klärungen, die wichtig sind, wenn Antwort auf die Frage gegeben werden soll: wie bewerten wir sittlich-moralisch eine gegebene Situation?

Es sei die Reflexion über die Würde des Menschen mit einer Schrift eingeleitet, die für das Denken jener Epoche charakteristisch ist, die wir Renaissance nennen. In dieser Epoche, deren Beginn in der zweiten Hälfte des 14. Jahrhunderts lag, trat die Frage nach der Würde des Menschen in das Zentrum der philosophischen und theologischen Anthropologie; und die gegebenen Deutungsversuche haben das Denken über diesen Topos bis zum heutigen Tage beeinflusst. Mir liegt die nun aufzurufende Schrift nicht nur wegen ihrer historischen Bedeutung, nicht nur wegen ihrer auch heute noch höchst relevanten Aussagen 
am Herzen, sondern auch wegen der Tatsache, dass sie mir später die Möglichkeit eröffnen wird, das angesprochene Werk von Johann Sebastian Bach - und hier vor allem das Präludium und die Fuge in cis-Moll - in meine Überlegungen aufzunehmen.

Es handelt sich um die vom Florentiner Gelehrten Pico della Mirandola verfasste Schrift „De hominis dignitate“ (deutsch: „Über die Würde des Menschen“), die im Jahre 1496 veröffentlicht wurde (Mirandola 1990). Pico leitet diese Schrift mit folgenden Aussagen ein, die die Fähigkeit des Menschen zur Selbstgestaltung und Weltgestaltung genauso wie das Geschenk der Freiheit in das Zentrum rücken (1990, S. 6 f.):

„Endlich beschloss der höchste Künstler, dass der, dem er nichts Eigenes geben konnte, Anteil habe an allem, was die Einzelnen jeweils für sich gehabt hatten. Also war er zufrieden mit dem Menschen als Geschöpf von unbestimmter Gestalt, stellte ihn in die Mitte der Welt und sprach ihn so an: ,Wir haben dir keinen festen Wohnsitz gegeben, Adam, kein eigenes Aussehen noch irgendeine besondere Gabe, damit du den Wohnsitz, das Aussehen und die Gaben, die du selbst dir ausersiehst, entsprechend deinem Wunsch und Entschluss habest und besitzest. Die Natur der übrigen Geschöpfe ist fest bestimmt und wird innerhalb von uns vorgeschriebener Gesetze begrenzt. Du sollst dir deine ohne jede Einschränkung und Enge, nach deinem Ermessen, dem ich dich anvertraut habe, selber bestimmen. Ich habe dich in die Mitte der Welt gestellt, damit du dich von dort aus bequemer umsehen kannst, was es auf der Welt gibt. Weder haben wir dich himmlisch noch irdisch, weder sterblich noch unsterblich geschaffen, damit du wie dein eigener, in Ehre frei entscheidender, schöpferischer Bildhauer dich selbst zu der Gestalt ausformst, die du bevorzugst."

Jeder Mensch besitzt Würde; diese ist nicht an Eigenschaften, nicht an Leistungen gebunden. Sie ist a priori gegeben. Jeder Mensch hat zudem eine Vorstellung von seiner Würde, das heißt, er stellt implizit oder explizit Kriterien auf, die erfüllt sein müssen, damit ihm das eigene Leben als ein würdevolles erscheint. In dem Beitrag von Pico della Mirandola (1990) ist nun ausdrücklich auch die Verwirklichung von Würde angesprochen, das heißt, es wird eine Bedingung genannt, unter der die Würde des Menschen lebendig wird. Diese Bedingung lautet: die Möglichkeit, oder besser: die Freiheit zur Selbstgestaltung und Weltgestaltung. Diese Freiheit zur Selbstgestaltung und Weltgestaltung ist zugleich eine Aufgabe, die dem Menschen gestellt ist: nur wenn er diese Aufgabe löst, kann sein Leben - in Freiheit - gelingen, verwirklicht er seine Würde. Die Freiheit zeigt sich auch in persönlichen Vorstellungen von Würde, die jeder Mensch vertritt (und die immer in ihrer gesellschaftlichen und kulturellen Mitbedingtheit betrachtet werden müssen); diese persönlichen Vorstellungen spiegeln 
sich in den Kriterien eines guten Lebens wider, die die Person auch für sich selbst definiert: diese Kriterien müssen erfüllt sein, damit in ihren Augen das Leben ein stimmiges und sinnerfülltes ist, damit das Leben gelingt.

Schon an dieser Stelle sei der Bezug zur Künstlichen Intelligenz hergestellt: Inwiefern berühren, um nur ein Beispiel für Künstliche Intelligenz zu wählen, Roboter, die stellvertretend für eine Person bestimmte Aktionen ausüben, damit auch die Würde des Menschen? Diese Frage ist deswegen so wichtig, weil mit Künstlicher Intelligenz immer auch Fragen der Autonomie, der Freiheit des Menschen berührt sind: gibt dieser seine Freiheit - in Teilen - auf, wenn er sich der Robotertechnologie anvertraut? Wer ist nun in letzter Hinsicht der für das Handeln Verantwortung Übernehmende: die Person oder der Roboter? An dieser Stelle sei schon eine Aussage getroffen, die in der jüngst erstellten Stellungnahme des Deutschen Ethikrates (2020) zentrale Bedeutung annimmt: die Künstliche Intelligenz muss daraufhin angelegt sein, dass sie die Person darin unterstützt, ihr Leben, ihre Welt zu gestalten, dass sie also in ihren Aktionen in letzter Konsequenz eine Stellvertreterposition einnimmt: sie stellt sich in den Dienst der Person, alle Aktionen, die sie ausführt, müssen die sittlich-moralischen Haltungen der Person als Ausgangspunkt und Grundlage wählen. In diesem Falle fügt sich die Robotertechnologie - so zum Beispiel im Kontext der Pflege - in das grundlegende Bedürfnis des Menschen nach Selbstgestaltung und Weltgestaltung ein, sie wird in das individuelle Handlungsrepertoire geradezu inkorporiert. Sie kann zugleich als Erweiterung dieses Handlungsrepertoires betrachtet werden, ohne sich dabei von den sittlich-moralischen Haltungen der Person zu lösen.

Damit ist auch schon klar zum Ausdruck gebracht: die Künstliche Intelligenz steht nicht a priori der Freiheit, der Selbstgestaltung, der Weltgestaltung der Person entgegen; sie beschneidet nicht a priori die Würde des Menschen. Ein derartiger Technik-Skeptizismus wäre auch alles andere als hilfreich für eine tiefer gehende anthropologische Diskussion. Vielmehr muss es um die Frage gehen, inwieweit bei der Entwicklung und Konstruktion, inwieweit bei der Applikation von Robotertechnologie immer mitgedacht wird, dass diese die Person in ihrer Selbstgestaltung und Weltgestaltung unterstützt und ihr auch - vielleicht sogar gerade in Phasen deutlich erhöhter Verletzlichkeit - Möglichkeiten bietet, einen signifikanten Anteil der Entscheidungs- und Handlungskompetenz aufrechtzuerhalten und zu verwirklichen: ein großartiger Beitrag zur Selbstgestaltung und Weltgestaltung.

Die Selbstgestaltung und Weltgestaltung im Alter bilden nun Gegenstand meiner Überlegungen: wie drücken sich Selbstgestaltung und Weltgestaltung aus? 


\section{$2 \quad$ Selbstgestaltung}

Die Selbstgestaltung zeigt sich zunächst in der vertieften, konzentrierten Auseinandersetzung des Individuums mit dem eigenen Selbst. Ich wähle den Terminus Introversion mit Introspektion (Kruse 2017), um diese konzentrierte Auseinandersetzung differenziert zu umschreiben. Im Zentrum steht das Selbst, das in der psychologischen Forschung als dynamischer Kern der Persönlichkeit betrachtet wird (Brandtstädter 2007). Das Selbst integriert alle Erlebnisse, Erfahrungen und Erkenntnisse, die das Individuum im Laufe seines Lebens in der Begegnung mit anderen Menschen, in der Auseinandersetzung mit der Welt, aber auch in der Auseinandersetzung mit sich selbst und seiner Biografie gewinnt. In dem Maße, in dem Menschen offen sind für neue Erlebnisse, Erfahrungen und Erkenntnisse, entwickelt sich auch das Selbst weiter: Dieses zeigt sich gerade in der Verarbeitung neuer Erlebnisse, Erfahrungen und Erkenntnisse in seiner ganzen Dynamik, in seiner (schöpferischen) Veränderungskapazität. Die Umschreibung Introversion mit Introspektion wähle ich, um die besondere Sensibilität alter Menschen für alle Prozesse hervorzuheben, die sich in ihrem Selbst vollziehen (Staudinger 2015). - Hier spielt auch der Lebensrückblick - der in der Theorie von Erik Homburger Erikson (1998) einen bedeutenden Teil der Ich-Integrität im Alter bildet - eine wichtige Rolle. Dieser Lebensrückblick betrifft in zentraler Weise das Selbst: Inwieweit werden dem Individuum bei dieser Spurensuche noch einmal Aspekte seines Selbst bewusst, die dieses aus heutiger Sicht positiv bewertet, inwieweit Aspekte des Selbst, die dieses eher negativ bewertet (Butler 1963)? Inwieweit gelingt es dem Individuum trotz negativer Bewertungen, sich selbst Freund zu sein, die eigene Biografie in ihren Höhen und Tiefen als etwas anzunehmen, das in eben dieser Gestalt stimmig, sinnerfüllt, notwendig war, inwieweit kann das Individuum sich selbst, aber auch anderen Menschen im Rückblick vergeben (Ritschl 2004)? Schließlich stößt die begrenzte Lebenszeit Prozesse der Introversion mit Introspektion an: In der Literatur wird auch von Memento-mori-Effekten gesprochen (Brandtstädter 2014), womit Einflüsse der erlebten Nähe zum Tod auf das Selbst gemeint sind. Im Zentrum stehen eine umfassendere Weltsicht und eine damit einhergehende Ausweitung des persönlich bedeutsamen Themenspektrums, weiterhin eine gelassenere Lebenseinstellung, begleitet von einer abnehmenden Intensität von Emotionen wie Ärger, Trauer, Reue und Freude. Zudem treten Spiritualität, Altruismus und Dankbarkeit stärker in das Zentrum des Erlebens (Kruse und Schmitt 2018). 
Ein weiteres bedeutsames Merkmal der Selbstgestaltung ist die Offenheit: Die konzentrierte, vertiefte Auseinandersetzung mit sich selbst wird durch die Offenheit des Individuums für neue Eindrücke, Erlebnisse und Erkenntnisse gefördert. Offenheit wird in der psychologischen Literatur auch mit dem Begriff der kathektischen Flexibilität (Peck 1968) umschrieben, was zum einen bedeutet, dass auch neue Lebensbereiche emotional und geistig besetzt und damit subjektiv thematisch werden, was zum anderen bedeutet, dass sich Menschen auf neue Handlungsstrategien einlassen: hier übrigens kommt auch die Künstliche Intelligenz ins Spiel. Inwieweit, so lässt sich fragen, ist das Individuum offen für die Nutzung der Künstlichen Intelligenz, also zum Beispiel für die Integration robotischer Systeme in den Lebensraum und Alltag? Der Grad der Offenheit für neue Erlebnisse, Erfahrungen und Handlungsstrategien - mithin auch für innovative technische Erzeugnisse - ist weniger vom Lebensalter der Person als von der seelisch-geistigen Flexibilität bestimmt, die diese in der Biografie entwickelt und kultiviert hat. Aus diesem Grunde lässt sich auch mit Blick auf die Künstliche Intelligenz feststellen, dass die digitale Spaltung eher an Sozialschicht-Grenzen und weniger an Alters-Grenzen thematisch wird (Kommission 2020). Offenheit bedeutet in einem umfassenderen Sinne, dass sich das Individuum auf den fließenden Charakter des Selbst einlässt und damit auch etwas Neues hervorbringt, schöpferisch lebt. Wir verdanken Friedrich Nietzsche (1844-1900) - nämlich seiner 1878 anlässlich des 100. Todestages Voltaires erschienenen Schrift „Menschliches, Allzumenschliches - ein Buch für freie Geister" - ein bemerkenswertes Zitat, das den fließenden Charakter des Selbst, das schöpferische Leben anschaulich umschreibt:

\footnotetext{
„Wer nur einigermaßen zur Freiheit der Vernunft gekommen ist, kann sich auf Erden nicht anders fühlen denn als Wanderer - wenn auch nicht als Reisender nach einem letzten Ziele: denn dieses gibt es nicht. Wohl aber will er zusehen und die Augen dafür offen haben, was alles in der Welt eigentlich vorgeht; deshalb darf er sein Herz nicht allzu fest an alles Einzelne anhängen; es muss in ihm selber etwas Wanderndes sein, das seine Freude an dem Wechsel und der Vergänglichkeit habe." (Nietzsche 1878/1998, S. 65)
}

Das Potenzial zur Selbstgestaltung ist nicht mit einem gewissen Alter abgeschlossen, sondern besteht - sofern nicht schwere Krankheiten dieses Potenzial zunichtemachen - bis zum Ende des Lebens. 


\section{$3 \quad$ Weltgestaltung}

In einer ersten Annäherung ist mit Weltgestaltung die Schaffung einer sozialen, räumlichen und technischen Umwelt gemeint, die den eigenen Ansprüchen an Stimulation, Bezogenheit und Teilhabe, Privatsphäre, Intimität, Ästhetik und Barrierefreiheit entspricht. Dieses hier zum Ausdruck kommende, grundlegende Bedürfnis nach Weltgestaltung ist nicht nur in jüngeren, sondern auch in späteren Lebensphasen deutlich erkennbar; in ihm drückt sich auch die Identität der Person aus. Die Integration robotischer Systeme lässt sich auch vor dem Hintergrund des Motivs der Weltgestaltung deuten: inwieweit trägt diese Integration dazu bei, dass das Individuum in einer sozialen und räumlichen Umwelt lebt, dies es meistern kann, in der sich der Teilhabegedanke verwirklicht, in der Lebensqualität erfahrbar wird?

Ein Beispiel für die Roboter-Technologie, das hier angeführt werden soll, bildet das Exoskelett, welches einem Schlaganfallpatienten hilft, trotz einer (noch) nicht zu lindernden Hemiparese eine deutlich höhere Mobilität zu zeigen. Das Exoskelett leitet einzelne Bewegungsabläufe autonom ein, wobei es sich aber in den Gesamtbewegungsablauf einschwingt bzw. einschmiegt. Für eine Patientin bzw. einen Patienten bedeutet das Anlegen eines Exoskeletts zunächst einen erheblichen Eingriff in ihre bzw. seine Autonomie. Denn einzelne Bewegungsabläufe werden vorgegeben, andere Bewegungsabläufe werden korrigiert. Zugleich aber wird mit dem Exoskelett die Weltgestaltung erheblich gefördert: die Person kann sich nun in stärkerem Maße selbstständig in ihrer Umwelt bewegen, was nicht nur eine bessere Erreichbarkeit physikalischer Orte, sondern auch sozialer Orte bedeutet.

Bereits dieses Beispiel zeigt: wenn über den Wert von Künstlicher Intelligenz gesprochen wird, so ist diese immer auch im Kontext von Weltgestaltung zu betrachten: inwiefern ermöglicht oder fördert diese die Kontrolle über die räumliche Umwelt, inwieweit ermöglicht oder fördert sie den Austausch mit der sozialen Umwelt, inwieweit hilft sie, Umweltbereiche (und damit auch Lebensbereiche) zu erschließen, die der Person andernfalls (weitgehend) verschlossen blieben? Die Bewertung von Künstlicher Intelligenz kann also nie eine abstrakte, von den individuellen Kompetenzen und Bedürfnissen losgelöste sein: entscheidend ist, in welcher Weise sie hilft, im konkreten Fall das Bedürfnis nach Weltgestaltung zu verwirklichen. Gerade damit ist das Zusammenwirken von menschlicher und künstlicher Intelligenz angesprochen. Durch dieses Zusammenwirken wird das Individuum bei der Verwirklichung seines Bedürfnisses nach Weltgestaltung unterstützt. 
In einer zweiten Annäherung ist mit Weltgestaltung die Sorge der Person gemeint, in der sich zunächst das Bedürfnis des Menschen nach praktizierter Mitverantwortung ausdrückt, also der Wunsch, etwas für andere Menschen zu tun, deren Entwicklung zu fördern (Kruse 2017; Kruse und Schmitt 2015, 2016). Sorge meint zudem die Sorge, die die Person von anderen erfährt. Dabei ist auch mit Blick auf Sorgebeziehungen im hohen Alter hervorzuheben, wie wichtig ein Geben und Nehmen von Hilfe und Unterstützung (Reziprozität) für die Akzeptanz erfahrener Sorge ist. Die fehlende Möglichkeit, die empfangene Sorge zu erwidern, macht es schwer, Sorge anzunehmen. Dieser Aspekt gewinnt besondere Bedeutung in Phasen erhöhter Verletzlichkeit. Gerade in solchen Phasen sind Menschen sensibel dafür, ob sie primär als Hilfeempfangende wahrgenommen und angesprochen werden, oder ob sie auch in ihrer Kompetenz, selbst Hilfe und Unterstützung zu leisten, ernst genommen werden. Zugleich ist im thematischen Kontext von Sorge immer mitzudenken, wie wichtig es ist, dass das Individuum rechtzeitig lernt, Hilfe und Unterstützung, die objektiv nötig ist, bewusst anzunehmen (Baltes 1996; Kruse 2005a). - Mit dem Begriff der Sorge ist nicht allein das Wohl einzelner Menschen angesprochen, für die das Individuum Mitverantwortung übernimmt, sondern auch das Wohl der Welt. Damit tritt die politische Dimension in das Zentrum meiner Argumentation. Mit dem politischen (und nicht nur psychologischen) Verständnis von Sorge folge ich den politikwissenschaftlichen Beiträgen von Hannah Arendt (1993), die ausdrücklich von der Liebe zur Welt (Amor mundi) spricht und diese als einen wichtigen Grund für ihre Arbeit an einer politischen Theorie nennt - so lesen wir in einem ihrer Briefe an Karl Jaspers. Die Liebe zur Welt führt nach Hannah Arendt zur Sorge um die Welt, die den Kern, den Mittelpunkt der Politik bildet. Hannah Arendt löst ihre Deutung von Welt nie vom Menschlichen ab. Wenn sie von Welt spricht, so orientiert sie sich grundsätzlich am Menschlichen - nämlich an einem öffentlichen Raum, in dem sich das Zwischen den Menschen entfalten kann, in dem sich Menschen in Wort und Tat begegnen, die Gestaltung der Welt als eine gemeinsam zu lösende Aufgabe begreifen. Und Hannah Arendt geht noch weiter: Ihr Verständnis von Politik orientiert sich auch an dem Wesen der Freundschaft (Arendt 1989). Inwiefern? Sie hebt hervor, dass das Schließen von Freundschaften keinem äußeren Zweck geschuldet ist, sondern dass dieses hervorgeht aus der Erfahrung des Zwischen, in dem sich Menschen im Vertrauen darauf zeigen und aus der Hand geben können, dass sie in ihrer Unverwechselbarkeit erkannt und angenommen werden - dieses Vertrauen ist dabei entscheidend für die Initiative, für den Neubeginn, für die Gebürtlichkeit (Natalität) des Menschen.

Welche Konsequenzen ergeben sich aus diesem psychologischen, anthropologischen und politikwissenschaftlichen Verständnis von Sorge für die ethischen 
Fragen der Künstlichen Intelligenz? Auch hier soll ein Beispiel angeführt werden, das hilft, diese Konsequenzen zu veranschaulichen. Ich denke an die robotischen Monitor-Techniken, die sich eigenständig oder von der Nutzerin bzw. dem Nutzer ferngesteuert in der Wohnung bewegen können. Ich denke weiterhin an Telepräsenzsysteme, die die Überwachung von Körperfunktionen durch medizinische oder pflegerische Fachpersonen ermöglichen; zugleich sind sie für Interaktionen mit nahestehenden Personen funktional, die weit entfernt leben und mit denen der unmittelbare (face-to-face-) Austausch nicht möglich ist. $\mathrm{Zu}$ nennen sind schließlich Roboter, die der sozialen Begleitung dienen (Sharkey und Sharkey 2012); diese robot companions werden zudem in ihren Potenzialen zur Unterstützung therapeutischer Maßnahmen in Psychiatrie, Psychologie und Psychotherapie diskutiert (Fiske et al. 2019). Mit derartigen Robotertechniken kann zunächst dazu beigetragen werden, dass gesundheitlich belastete und funktionell eingeschränkte Personen länger in ihrem Privathaushalt verbleiben können: dies hat nicht nur Folgen für die Selbstgestaltung, sondern auch und vor allem für die Weltgestaltung, da die Person nicht ihre vertraute soziale und räumliche Umwelt aufgeben muss. Zudem können diese Robotertechniken die bewusst angenommene Abhängigkeit von Hilfen durch andere Menschen fördern. Warum? Indem bestimmte Hilfeleistungen einem Roboter übertragen werden, wird vermieden, dass die angestrebte Reziprozität von Hilfen in den nahestehenden Beziehungen mehr und mehr erodiert. Dies könnte der Fall sein, wenn pflegende Angehörige zahlreiche Hilfen zu erbringen haben, die in ihrer Häufung als (zusätzliche) Belastung empfunden werden. Im Falle zurückgehender Reziprozität kann es für die pflegebedürftige Person schwer sein, die Abhängigkeit von der Hilfe anderer Menschen bewusst anzunehmen. Und schließlich darf nicht der potenzielle Gewinn für die Weltgestaltung übersehen werden, der durch Telepräsenzsysteme geschaffen wird: der Zugang zur Welt ist damit auch jenen Menschen eröffnet, die ihre Wohnung - aufgrund schwerer Einbußen ihrer Mobilität - gar nicht mehr oder nur unter größten Kraftanstrengungen verlassen können. Indem der Zugang zur Welt eröffnet ist, kann sich nicht nur das soziale, sondern auch das politische Interesse der Person verwirklichen.

In einer dritten Annäherung ist mit Weltgestaltung die Wissensweitergabe, ist auch das Fortwirken des Individuums in nachfolgenden Generationen angesprochen (in der Begrifflichkeit von Hannah Arendt (1960): symbolische Unsterblichkeit). Dieses Fortwirken vollzieht sich auch auf dem Wege materieller und ideeller Produkte, die das Individuum erzeugt und mit denen es einen Beitrag zum Fortbestand und zur Fortentwicklung der Welt leistet (Staudinger 1996). So sehr eine Person in der Erinnerung an das gesprochene Wort und die einmalige Gebärde fortlebt, so sehr Begegnungen mit dieser in uns emotional und 
geistig fortwirken, so wichtig ist es auch, die materiellen und ideellen Produkte im Auge zu haben, die sich nicht notwendigerweise unmittelbaren Begegnungen mit nachfolgenden Generationen verdanken, sondern die in Verantwortung vor der Welt und für die Welt entstanden sind. Auch diese Produkte hat Hannah Arendt im Auge, wenn sie von symbolischer Unsterblichkeit spricht (Arendt 1960). Es geht hier um Werke, die (auch) aus einer Verantwortung gegenüber der Welt entstanden sind, mit denen bewusst zum Fortbestand und zur Fortentwicklung der Welt beigetragen werden soll (Blumenberg 1986). Das Handeln als höchste Form der Vita activa beschränkt sich also nicht allein auf den unmittelbaren, konkreten Austausch mit Menschen. Wir treten auch in unseren Gedanken in einen - vielleicht virtuell zu nennenden - Austausch mit Menschen, die wir kannten (und die heute nicht mehr leben), die wir kennen (denen wir aber gegenwärtig nicht unmittelbar begegnen können) und die wir noch nicht kennen, ja, niemals kennenlernen werden: Damit ist in besonderer Weise die geistige Dimension der Vita activa, des gemeinsamen Handelns (als eines Konstituens der Vita activa) und des Politischen (als der Umschreibung von gemeinsam geteilter Verantwortung vor der Welt und für die Welt) angesprochen.

Die Kommunikationstechnologie erscheint geradezu wie gemacht für diese Wissensweitergabe. Telepräsenzsysteme können die Wissensweitergabe in einer sehr lebendigen Art und Weise sicherstellen, weil sie eine vergleichsweise enge Berührung von Sender und Empfänger ermöglichen. Sie können schließlich dazu beitragen, dass wir an dem Leben einer Person teilhaben, die in ihren Kommunikationsmöglichkeiten erheblich beeinträchtigt, zugleich aber von dem Motiv geleitet ist, das eigene - lebendige - Wissen an andere Menschen weiterzugeben, sie an dem eigenen Schicksal teilhaben zu lassen. Pathemata paideumata genesetai tois allois (deutsch: mein Leiden, so schwer es ist, kann auch zu Lehren für Andere werden: eine Aussage, die sich der altgriechischen Philosophie verdankt, kann durch technische Entwicklungen in besonderer Weise verwirklicht werden.

\section{$4 \quad$ Verletzlichkeit}

Wie ist Verletzlichkeit im hohen Alter zu verstehen, durch welche Merkmale zeichnet sich diese aus? Vor dem Hintergrund der mittlerweile umfangreichen empirischen Literatur zum hohen Alter ist davon auszugehen, dass sich im Verlauf des neunten Lebensjahrzehnts der Übergang vom höheren (dritten) zum hohen (vierten) Alter allmählich, fließend, kontinuierlich vollzieht (Kruse 2017). Dabei ist das neunte Lebensjahrzehnt nicht als ein Jahrzehnt zu begreifen, in dem 
körperliche und psychische Erkrankungen notwendigerweise plötzlich, abrupt über das Individuum hereinbrechen. Vielmehr ist im neunten Lebensjahrzehnt eine graduell zunehmende Anfälligkeit des Menschen für neue Erkrankungen und funktionelle Einbußen ebenso erkennbar wie die graduelle Zunahme in der Schwere bereits bestehender Erkrankungen und bereits bestehender funktioneller Einbußen (Fried et al. 2001). Damit ist ein wichtiges Merkmal des hohen Alters beschrieben, das auch im Erleben der Menschen dominiert: Die allmählich spürbare Zunahme an Krankheitssymptomen, die allmählich spürbaren Einbußen in der körperlichen, zum Teil auch in der kognitiven Leistungsfähigkeit, schließlich die allmählich spürbaren Einschränkungen in alltagsbezogenen Fertigkeiten werden vom Individuum im Sinne der erhöhten Verletzlichkeit erlebt und gedeutet (Clegg et al. 2013). Verletzlichkeit heißt dabei nicht Gebrechlichkeit; letztere ist vielmehr Folge ersterer. Verletzlichkeit lässt sich auch nicht mit den medizinischen Begriffen Multimorbidität und Polysymptomatik angemessen umschreiben. Vielmehr meint Verletzlichkeit eine erhöhte Anfälligkeit und Verwundbarkeit, mithin das deutlichere Hervortreten von Schwächen, meint verringerte Potenziale zur Abwehr, Kompensation und Überwindung dieser körperlichen und kognitiven Schwächen. Die objektiv messbare wie auch die subjektiv erlebte Verletzlichkeit tritt zu interindividuell unterschiedlichen Zeitpunkten im neunten Lebensjahrzehnt auf; sie kann sich bei dem einen sogar noch später (also erst im zehnten Lebensjahrzehnt), bei dem anderen sogar noch früher (also schon im achten Lebensjahrzehnt) einstellen. Entscheidend ist, dass im Verlauf des neunten Lebensjahrzehnts bei der Mehrzahl alter Menschen eine derartige erhöhte Verletzlichkeit objektiv nachweisbar ist und subjektiv auch als eine solche empfunden wird.

Mit dem Hinweis auf die erhöhte Verletzlichkeit wird angedeutet, dass im hohen Lebensalter ein Merkmal der Conditio humana - nämlich die grundsätzliche Verwundbarkeit - noch einmal stärker in das Zentrum tritt, dabei auch in das Zentrum des Erlebens. Mit diesem Hinweis wird die vielfach vorgenommene, strikte Trennung zwischen drittem und viertem Lebensalter relativiert: Es ist nicht so, dass das dritte Lebensalter ganz unter dem Zeichen erhaltener körperlicher, kognitiver und sozio-emotionaler Kompetenz, das vierte Lebensalter hingegen ganz unter dem Zeichen verloren gegangener körperlicher, kognitiver und sozio-emotionaler Kompetenz (im Sinne eines modus deficiens) stünde. Vielmehr finden wir auch im dritten Alter graduelle Verluste und damit allmählich stärker werdende Schwächen, die in summa auf eine erhöhte Verletzlichkeit des Menschen deuten; und im vierten Alter sind vielfach seelische, geistige, sozio-emotionale und sozial-kommunikative Ressourcen zu beobachten, die das Individuum in die Lage versetzen, ein schöpferisches, persönlich sinnerfülltes 
und stimmiges Leben zu führen - dies auch in gesundheitlichen Grenzsituationen (Brothers et al. 2016).

Die Robotertechnologie möchte auch Antwort auf die Frage geben, wie sie die Person in Phasen deutlich erhöhter Verletzlichkeit unterstützen kann, sodass ihr Selbstgestaltung und Weltgestaltung auch in Phasen dieser erhöhten Verletzlichkeit möglich sind. Ein überzeugendes Beispiel der Unterstützung ist eine neurochirurgische Intervention, die auch mit dem Terminus der Tiefen Hirnstimulation belegt wird. Durch ein chirurgisch hergestelltes Loch in der Schädeldecke können elektrische Sonden in das Gehirn eingesetzt werden: es wird jene Hirnregion gewählt, in der die Funktion der Nervenzellen unterbrochen oder bleibend gestört ist (Bei Patienten*innen mit einer ParkinsonErkrankung bildet die Substantia nigra die geschädigte Region.). Die Elektroden sind mit einem unter das Schlüsselbein implantiert Schrittmacher verbunden. Schwache Stromstöße bedingen eine kontinuierliche elektrische Reizung jener Hirnregion, die die überaktiven Nervenzellen hemmt. Die Beweglichkeit verbessert sich erheblich: Die Tiefe Hirnstimulation im fortgeschrittenen Stadium einer Parkinson-Erkrankung ist deutlich besser als die alleinige Behandlung mit Medikamenten (L-Dopa) zu einer Symptomlinderung geeignet, auch wenn keine umfassende Heilung von der Erkrankung versprochen werden kann. Zudem sind Mensch-Maschine-Verbindungen geeignet, elektrophysiologische Signale aus einzelnen Hirnregionen in andere Hirnregionen zu übertragen: zu nennen ist hier die computergestützte Übertragung von Kognitionen an motorische Zentren bei Patienten*innen, die an einer fortgeschrittenen Amyotrophen Lateralsklerose leiden. Diese Krankheit gehört zur Gruppe der Motoneuronkrankheiten: das motorische Nervensystem erkrankt sowohl in seinen zentralen (oberes Motoneuron, Pyramidenbahnen bis Rückenmark) als auch in seinen peripheren Anteilen (unteres Motoneuron, Hirnstamm und Rückenmark mit den motorischen Nervenfasern bis zum Muskel). Mit einer Computervorrichtung, die die Gedanken dechiffrieren kann, wird versucht, die Gedanken in Impulse zu übertragen, die an die verschiedenen Muskelpartien weitergeleitet werden. Dadurch erhält die nicht-geschädigte Muskulatur Impulse, die ihrerseits die Ausführung und Koordination von Bewegungen anstoßen sowie die Muskulatur erregen, wodurch Steifheit gelindert werden kann. Das Exoskelett, das um Beine, Becken und Rücken, Arme gelegt werden kann, dient dazu, Funktionsabläufe stellvertretend für das geschädigte periphere Organ auszuführen; zudem wird versucht, durch gezielte Erregungsmuster die Kontrollinstanzen im Gehirn (motorische Zentren) gezielt zu erregen. 
Wo liegen hier ethische Herausforderungen, wo ethische Dilemmata?

Die Eingriffe, die in den Organismus vorgenommen werden, sind erheblich. Verbunden damit ist aufseiten der Patienten*innen die Sorge, nicht mehr Herr im eigenen Hause zu sein, d. h. Kontrolle über Funktionen abzugeben. Allerdings steht dieser Sorge ein Gewinn gegenüber: nämlich auf dem Wege eines technischen Hilfsmittels vermehrt Kontrolle über Körperfunktionen ausüben zu können. Die Beispiele zeigen, wie wichtig es ist, Patienten*innen Künstliche Intelligenz und deren Effekte mit Blick auf ihre spezifische Erkrankung und ihre spezifischen Funktionseinbußen zu erklären. Der Begriff des Arbeitsbündnisses ist in diesem Kontext besonders wichtig. Denn nur auf der Grundlage eines Arbeitsbündnisses wird es gelingen, die hier sehr kurz beschriebenen potenziellen Effekte tatsächlich zu erzielen, in denen sich auch das Zusammenwirken von menschlicher und künstlicher Intelligenz eindrucksvoll zeigt.

\section{Johann Sebastian Bach - ein kurzer Blick auf seine Biografie}

Ich hatte zu Beginn des Beitrags angedeutet, dass auch eine Reflexion über Präludium und Fuge in cis-Moll, Wohltemperiertes Klavier, Band I, von Johann Sebastian Bach (1685-1750) erfolgen wird. Das Präludium leitet im Verständnis des Komponisten die Fuge ein: aus diesem Grunde wird auch von einem Prä-ludium, einem Vor-spiel gesprochen. In den beiden Bänden Wohltemperiertes Klavier, I und II finden sich jeweils 24 Satzpaare aus einem Präludium und einer Fuge in allen Dur- und Molltonarten. Die Satzpaare sind dabei chromatisch aufsteigend angeordnet (von C-Dur bis h-Moll).

Warum erfolgt hier dieser Einschub? In dem Vortrag, auf dem dieser Beitrag aufbaut, hatte ich die Ehre, Präludium und Fuge in cis-Moll, Wohltemperiertes Klavier, Band I, vorzutragen. Die Überlegung des Veranstalters war, eine Reflexion über das hohe Lebensalter auch auf musikalischem Wege vorzunehmen: und es wird deutlich werden, dass uns eine Fuge sehr viel über das hohe Lebensalter mitzuteilen vermag.

Zunächst seien einige wenige Aussagen über die Biografie von Johann Sebastian Bach getroffen; danach sei auf eine grundlegende Merkmale der Fugenkomposition eingegangen; hier vor allem auf die Fuge in cis-Moll.

Johann Sebastian Bach zeigte in allen Phasen seiner Biografie ein hohes Maß an Fleiß, an Offenheit, an schöpferischen Kräften (ausführlich in Wolff 2009). Schon im Kindes- und Schulalter begeisterte er sich für Musik - was auch damit zu tun hatte, dass im Hause der Eltern die Stadtpfeifer Eisenachs regelmäßig 
probten (Bachs Vater Ambrosius Bach leitete das Konsortium der Stadtpfeifer). Zugleich gehörte er in der Schule zu den Besten seines Jahrgangs. Im Alter von 15 Jahren brach er mit seinem Schulfreund Erdmann von Ohrdruf in Thüringen nach Lüneburg auf, um dort - im Michaeliskloster - um einen Freitisch (Stipendium) nachzusuchen; dieses Gesuch wurde angenommen, sodass Bach in der dortigen Schule mit 17 Jahren seine Matura ablegen konnte. In den weiteren Phasen seiner Biografie imponierte Bach nicht nur als Orgelprüfer und Organist (seine erste Anstellung erhielt er schon im Alter von 18 Jahren in Arnstadt), sondern auch und vor allem als Komponist, Orchester- und Chorleiter (stellvertretend für zahlreiche Ämter sei hier das Amt des Thomaskantors in Leipzig genannt). In seinen Kompositionen zeigt sich eine kaum zu überbietende Kreativität - nicht nur was den Umfang seines Oeuvres angeht, sondern auch dessen Qualität: Bach erfüllte nicht nur die damals bestehenden Maßstäbe höchster Kompositionskunst, sondern er setzte ganz neue Maßstäbe, so zum Beispiel mit der Missa in h-Moll, mit dem Musikalischen Opfer, mit der Kunst der Fuge - wobei hier nur drei Beispiele aus seinem Spätwerk angeführt wurden. Würde man auf seine mittlere Schaffensperiode Bezug nehmen, so wären die Johannes-Passion sowie die Matthäus-Passion als Beispiele für Kompositionen zu nennen, mit denen Bach Maßstäbe gesetzt hat. Neben dem schöpferischen Reichtum seiner Kompositionen (als hervorstechendem Merkmal seiner bis zum Lebensende bestehenden Kreativität) zeichnete sich Bach durch umfassende Bildung (so zum Beispiel in Philosophie und Theologie, in Mathematik, in Latein) sowie durch hohes Engagement für seine Schüler aus (er nahm noch in seinen letzten Lebensmonaten einen Schüler bei sich auf).

Zugleich war das Leben Bachs von zahlreichen, schweren und schwersten Belastungen bestimmt (ausführlich in Kruse 2014). Zu nennen sind der Verlust beider Elternteile im zehnten Lebensjahr (Johann Sebastian Bach wurde nach dem Tod seiner Eltern von seinem ältesten Bruder aufgenommen und lebte fünf Jahre bei diesem), der Tod seiner ersten Ehefrau Maria Barbara in seinem 36. Lebensjahr (wobei er nach der Rückkehr von einer sechswöchigen Konzertreise erfuhr, dass seine Frau verstorben und schon beerdigt sei und seine vier Kinder auf mehrere Familien aufgeteilt worden seien), der Tod von elf seiner 20 Kinder, die gesundheitlichen Einschränkungen in seinen letzten Lebensjahren und schließlich die Kränkung, dass bereits ein Jahr vor seinem Tod ein Nachfolger für ihn als Thomaskantor bestimmt wurde. Zudem musste sich Bach während seiner gesamten Berufstätigkeit immer wieder mit Kritik auseinandersetzen, die 
sich an seiner für die damalige Zeit höchst modernen Musik entzündete und ihm vor Augen führte, dass seine außerordentliche Begabung und Kreativität von geistlichen und weltlichen Oberen nicht erkannt wurde - eine Tatsache, die ihn schmerzte.

Aber in diesen Belastungssituationen war auch ein hohes Maß an psychischer Widerstandsfähigkeit (Resilienz) erkennbar, also die Fähigkeit, Belastungen verarbeiten und bewältigen zu können und in diesem Prozess schöpferische Kräfte $\mathrm{zu}$ entwickeln, die seinem Lebenswillen wie auch seiner Lebensgestaltung zugutekamen (ausführlich in Kruse 2015). Dabei wurde die Resilienz durch das Eingebundensein des Komponisten in unterschiedliche Ordnungen gefördert: In die Ordnung der Familie (Johann Sebastian Bach blickte auf befruchtende erste Lebensjahre zurück und auch nach dem Tod seiner Eltern fand er in der Familie Rückhalt), in die Ordnung der Musik, in die Ordnung des Glaubens (die den cantus firmus seiner Kompositionen bildete), in die Ordnung sozialer Beziehungen (hier ist vor allem die Mitverantwortung für nachfolgende Generationen - seine Kinder, Neffen, Schüler - zu nennen). Diese Ordnungen sollten sich über die gesamte Biografie als sehr stabil und damit haltgebend erweisen. Und schließlich entwickelte Johann Sebastian Bach schon früh Eigeninitiative, war immer offen für neue Eindrücke, zeigte in allen Lebensphasen großen Fleiß. Damit schuf er die Grundlage für seine außergewöhnliche Produktivität und Kreativität bis in die letzte Lebensphase.

In den letzten Lebensjahren litt Bach an den Folgen eines Diabetes mellitus Typ II, er verlor allmählich sein Augenlicht (die Erblindung war auch mitverursacht durch zwei völlig fehlgeschlagene Augenoperationen des Londoner Starstechers Taylor), er war aufgrund stark eingeschränkter Motorik schließlich nicht mehr in der Lage, seine Kompositionen selbst niederzuschreiben, sondern musste sich hierfür der Hilfe seiner Schüler bedienen. Kurz vor seinem Tod trat ein Schlaganfall auf. Und doch arbeitete er trotz dieser gesundheitlichen Einschränkungen an dem Musikalischen Opfer und schloss dieses ab, führte systematisch die Kunst der Fuge weiter, die zwar nicht vollständig niedergeschrieben werden konnte (der Contrapunctus 14 bricht nach Einführung des letzten Fugenthemas ab), die aber mit hoher Wahrscheinlichkeit in der Vorstellung Johann Sebastian Bachs fertiggestellt war, vollendete die h-Moll-Messe und schuf kurz vor seinem Tod den Choral Vor Deinen Thron tret ich hiermit (ausführlich in Kruse 2014). 


\section{$6 \quad$ Fuge in cis-Moll - Verbindung zu Alter, Sterben und Tod}

Der Begriff Fuge stammt aus der griechischen und lateinischen Sprache. Er beschreibt die Flucht. Flucht - phyge im Griechischen, fuga im Lateinischen - ist hier nicht im Sinne von Flüchten zu verstehen. Nein, Fuge meint etwas anderes, nämlich das Fliehen auf ein Ziel hin. In der lateinischen Sprache wird dieses Verständnis von fliehen mit dem Verb fugere umschrieben, das etwas anderes bedeutet als fugare, das mit in die Flucht schlagen übersetzt wird. Wir fliehen auf ein Ziel hin. Und was ist dieses Ziel? Im Verständnis von Johann Sebastian ist dieses Ziel der Tod, der nicht als Ende, sondern als Übergang verstanden wird.

Der Tod gilt in der Renaissance und in der Barockzeit - auch in der Kompositionslehre beider Epochen - als jener Punkt, auf den unser Leben zuläuft, als ein Punkt, auf den hin wir fliehen, was letzten Endes bedeutet, dass wir auch im Vorfeld des Todes den Aspekt der Selbstgestaltung und Weltgestaltung nicht vernachlässigen dürfen. Denn: wir sollen uns auf diesen Übergang vorbereiten und unsere nächsten Personen in diese Vorbereitung wie auch in das Abschiednehmen einbeziehen. Das Ende unseres Lebens ist somit nicht ein inferiorer Teil des Lebens, er ist nicht einer, der eigentlich nicht mehr zum Leben gehörte, von diesem abgeschnitten werden könnte. Nein: dieser Teil des Lebens ist ein bedeutender, in dem unsere Biografie zu einer gewissen Rundung kommen kann. Zudem - und hier kann man sich von der Philosophie des ägyptisch-römischen Neuplatonikers Plotin (205-270 n. Chr.) inspirieren lassen - findet am Ende unseres Lebens ein Übergang statt: nämlich jener der individuellen Seele in die Weltseele, jener des individuellen Geistes in den Weltgeist, schließlich der Rückgang des Seelischen und Geistigen in das Eine. An dieser Stelle sei angemerkt, dass der Kirchenvater Augustinus von Hippo (354430 n. Chr.) in seiner Konzeption von Seele und Geist auch auf die Lehre Plotins zurïckgriff. Und es gibt sehr gute Arbeiten zum Verständnis der Fuge von Johann Sebastian Bach, in denen dessen Fugenkompositionen in eine enge Beziehung zur Plotinschen Lehre des Sterbens als Rückkehr in die Heimat gesetzt werden (hier ist vor allem zu nennen: Dentler 2004; ein Überblick findet sich in Kruse, 2013).

Ein eindrucksvolles Beispiel für dieses Verständnis der Fuge im Denken von Johann Sebastian Bach lässt sich in seinem Werk Kunst der Fuge finden (siehe dazu Eggebrecht 1998). Im Contrapunctus 15 wird sein Name - B-A-C-H als drittes Fugenthema, das Johann Sebastian Bach über den Leitton cis zum Ton d führt, zum königlichen Ton. Er integriert also symbolisch seinen Namen in die göttliche Ordnung (Wolff 2009). Hier fühlen wir uns an einen eindrucksvollen Vers aus den Sonetten von Michelangelo Buonarroti - dieser lebte von 
1475-1564 - erinnert, wo es heißt: „Des Todes sicher, nicht der Stunde - wann. Das Leben kurz, nur wenig komme ich weiter, den Sinnen zwar scheint diese Wohnung heiter, der Seele nicht - sie bittet mich, stirb an" (Buonarroti 2002).

Noch einmal: wir streben auf ein großes Ziel hin - dieses Ziel ist unser Tod. Ich werde Ihnen gleich eine Fuge vorspielen, eine der großen Fugen des Johann Sebastian Bach: die Fuge in cis-Moll aus dem Wohltemperierten Klavier I. Am Ende einer jeden Fuge, die Johann Sebastian Bach komponiert hat, steht immer das musikalische Bild: Wir sind an dem Ziel angekommen. Dies ist auch und in besonderer Weise in der Fuge in cis-Moll der Fall. Bei Bach endet auch eine in Moll gesetzte Fuge mit einem Durakkord, was ausdrückt: Wir haben das Ziel erreicht. Zudem finden Sie in den letzten Takten einer Fuge eine Engführung aller Stimmen: die Stimmen setzen noch einmal nacheinander in kurzen Abständen ein und präsentieren das Thema der Fuge: in der cis-Moll-Fuge entsteht somit am Ende noch einmal eine unglaubliche Klangfülle und -dichte, wodurch der Zieloder Fluchtpunkt einmal mehr symbolisch ausgedrückt wird.

Und weiter: Die Fuge zeichnet sich dadurch aus, dass in der Vielfalt der Motive das Fugenthema (es können auch mehrere Fugenthemen sein) deutlich hervortritt. Im Verständnis von Johann Sebastian Bach lässt sich auch sagen: hier wird die göttliche Stimme in der Vielzahl der menschlichen Stimmen vernehmbar. Wir können, wenn wir dieses momentum specificum nicht theologisch, sondern psychologisch deuten wollen, auch sagen: in der Vielzahl der Motive wird ein zentrales Thema (oder: werden zentrale Themen) hörbar, Daseinsthemen, die für unser Erleben zentral sind. Diese Daseinsthemen rahmen die täglichen Erlebnisse und Erfahrungen sowie die Antworten auf diese ein.

Die Person ist immer auch von ihren Daseinsthemen her $\mathrm{zu}$ begreifen (Thomae 1968), die ihrerseits als zentrale Anliegen des Menschen verstanden werden können. Diese Themen ziehen sich als Leitmotive durch die Biografie, sie geben dem individuellen Dasein eine Kontinuität - auch wenn sie sich im Laufe der Biografie in ihrer Intensität, in ihrem Ausdruck wandeln. Eine biografische Analyse ist immer auch eine daseinsthematische Analyse (ausführlich in Kruse 2005b).

So können wir auch alten Menschen eine Fuge vorspielen und diese psychologisch wie theologisch erläutern. Und in dieser Erläuterung gelangen wir dann an den Punkt, an dem wir sagen: Erkennen Sie in den immer wiederkehrenden Themen auch eine Analogie zu jenen Themen, die Sie bereits seit langer Zeit beschäftigen, die für Ihr Erleben zentral waren, zentral sind?

Die cis-Moll-Fuge baut auf drei Themen auf, die immer in den fünf Stimmen aufgegriffen werden und sozusagen die Struktur der Fuge bilden. Das erste Thema der cis-Moll-Fuge erinnert an das dritte Thema des 15. Kontrapunkts 
der Kunst der Fuge: nämlich an das Thema B-A-C-H; in diesem ersten Thema ist ein Kreuzmotiv erkennbar, das auf eine religiöse Aussage dieser Fuge deutet.) Das zweite Thema ist ein in Achtel-Noten gesetzte Kantilene, die möglichst leicht und zart gespielt werden muss: hier wird in der Literatur auch gerne von der Verkörperung des Geistes gesprochen. Das dritte Thema ist in Viertel-Noten gesetzt, sehr pointiert zu spielen: hier wird in der Literatur auch gerne von der Verkörperung Gottes gesprochen. Kreuz Jesu Christi, Geist und Gott: wir stehen im Zentrum der musikalischen Umschreibung der Trinität.

Um diese drei Themen also gruppiert sich die gesamte Fuge. Die Fugenthemen müssen aus der Vielfalt der erklingenden Motive so deutlich herausgearbeitet werden, dass Sie diese an den verschiedenen Stellen der Fuge wiedererkennen - und damit die thematische Struktur, die den Kern dieser Fuge bildet. Am Ende des Stückes nehmen Sie eine Verdichtung wahr: alle Stimmen greifen jetzt diese drei Themen noch einmal in kurzen Abständen auf, sodass Sie den Eindruck gewinnen müssen (Sie können sich dessen gar nicht erwehren), dass hier eine Verdichtung, eine Engführung - wie man dies in der Musik nennt - stattfindet. Diese Verdichtung, diese Engführung: zeichnet diese nicht vielfach das menschliche Leben an dessen Ende aus? Das hohe und sehr hohe Lebensalter und das Ende des Lebens aus einer solchen Perspektive zu betrachten: dies erscheint mir als bedeutsam mit Blick auf ein tieferes Verständnis der Psyche alter Menschen sowie der Psyche sterbender Menschen.

\section{Prinzipien ethischer Bewertung digitaler Technologien}

Im Folgenden möchte ich auf die in biomedizinethischen Diskursen und ethischen Leitlinien durchscheinenden Prinzipien eingehen, die - auch wenn sich aus diesen keine verbindlichen Handlungsempfehlungen oder -entscheidungen ableiten lassen - im Sinne eines allgemeinen Orientierungsrahmens ethischer Entscheidungen zu verstehen sind, die mit Blick auf die Entwicklung, Verbreitung und Nutzung digitaler Technologien getroffen werden (European Commission 2018). Diese Prinzipien verweisen auf grundlegende Rechte der Person; die Beschneidung dieser Rechte hat in jedem Falle als (hinreichend informiert) zustimmungspflichtig bzw. im Falle eingeschränkter Entscheidungsfähigkeit als kompetent begründungs-, wenn nicht sogar genehmigungsbedürftig zu gelten. Denn es werden hier zentrale Aspekte der Selbstgestaltung und Weltgestaltung angesprochen; zudem stehen zentrale Aspekte der Identität, Intimität und Teilhabe im Zentrum. Aus den verschiedenen Prinzipien können sich konkurrierende 
Handlungsorientierungen ergeben, ethische Dilemmata also, die im Einzelfall auf der Grundlage von personenzentrierten Präferenzen und Kosten-Nutzen Erwägungen gelöst werden müssen. Gerade dann, wenn es um die personenzentrierten Präferenzen geht, stehen wir im Zentrum der Selbstgestaltung und Weltgestaltung.

Autonomy: Das Prinzip der Autonomie spricht die Fähigkeit der Person an, sich in Denken und Handeln an eigenen Wünschen und Präferenzen zu orientieren, bzw. die Freiheit, selbstgewählte Ziele und Pläne zu verfolgen: eine zentrale Dimension von Selbstgestaltung. Hierzu gehört ausdrücklich auch das Recht auf Verweigerung ohne vermeidbare Nachteile. Im Zusammenhang mit intelligenter assistierender Technologie bei Demenz umfasst Autonomie folgende Teilkomponenten:

a) Selbstständigkeit, und zwar im Sinne der Möglichkeit, auch unabhängig von kontingenten, das heißt für die Person nicht beeinflussbaren Grenzen und externer Gestaltung von Alltagsroutinen und Lebenswelt zu handeln: in meiner Diktion eine zentrale Dimension von Selbstgestaltung.

b) Altern in vertrauter Umgebung, und zwar im Sinne der Fähigkeit, im eigenen Haushalt und Quartier sicher, mit ausreichend hohem Standard und unabhängig zu leben, und dies unabhängig von Alter, Einkommen und Fähigkeitsniveau: in meiner Diktion eine zentrale Dimension von Weltgestaltung.

c) Nutzerzentrierung, und zwar im Sinne eines von den Bedürfnissen der zu unterstützenden Person ausgehenden und kontinuierlich anzupassenden und zu optimierenden Technikdesigns: in meiner Diktion eine zentrale Dimension von Selbstgestaltung und Weltgestaltung.

Dabei gilt die Autonomie der Person sowohl als ein zu respektierendes Recht als auch als eine aktiv zu fördernde Fähigkeit von potenziellen Nutzern. Besonders wichtig erscheint mir die Betonung der aktiv zu fördernden Fähigkeit des Individuums, eine Forderung, die sehr gut in den von Martha Nussbaum explizierten capability approach (fähigkeitsorientierten Ansatz) eines guten Lebens integriert werden kann (siehe Nussbaum 2011). Was hier deutlich wird und auch mit Blick auf die Robotertechnologie nicht genügend hervorgehoben werden kann: Sowohl Personen mit Assistenz- oder Pflegebedarf als auch Personen, die Assistenz oder Pflege leisten (bzw. die Verantwortung im Kontext der Rehabilitation wahrnehmen), müssen befähigt werden, Robotertechnologie in ihren möglichen Chancen und Risiken für die persönliche Lebenssituation zu bewerten und - vor allem - diese Technologie verantwortungsvoll zu nutzen. 
Und hier möchte ich direkt hinzufügen: die notwendige kognitive und Verhaltensplastizität ist bei alten Menschen, sofern bei ihnen keine neurokognitiven Erkrankungen vorliegen, vorhanden.

Privacy: Das Prinzip der Privatheit verweist auf die Fähigkeit und den Rechtsanspruch von Individuen und Gruppen, über den Kontakt und die Nähe zu Anderen (physical privacy) sowie über die Verfügbarkeit von Informationen über die eigene Person (informational privacy) selbstverantwortlich zu bestimmen: eine Bedingung für Selbstgestaltung und Weltgestaltung. Das Eindringen in die Intim- oder Privatsphäre der Person ist zu vermeiden und gegen deren Willen bzw. ohne deren Zustimmung auch gar nicht zu rechtfertigen. Des Weiteren ist die Sicherheit sensibler Daten unverzichtbare Voraussetzung des Einsatzes entsprechender Technik; Erhebung, Speicherung und Weitergabe personenbezogener Daten sind ohne ausdrückliche Zustimmung durch die Person zu vermeiden. Wenn diese Dimensionen von Privatheit nicht berücksichtigt werden und der durch sie konstituierte normative Rahmen nicht respektiert wird: dann wird die Person die durch die Robotertechnologie erbrachte Assistenz eher als einen Eingriff in die Privatsphäre denn als einen Beitrag zur Ermöglichung von Selbstgestaltung und Weltgestaltung begreifen. Die Integration von Robotertechnologie in diagnostische, therapeutische, rehabilitative und pflegerische Akte kann nur auf der Grundlage von geteilten Entscheidungen (shared decisions) zwischen jener Person, die auf Assistenz oder Pflege angewiesen ist, und jenen Personen, die diese leisten, vorgenommen werden.

Beneficence: Das Prinzip der Wohltätigkeit bzw. Wohlfahrt verweist auf die zentrale Zielsetzung kurativer Medizin. In der biomedizinischen Ethik besteht zunehmend Konsens, dass dieses Prinzip in einem umfassenden (holistischen) Sinne zu interpretieren ist, der über die Heilung von Erkrankungen im engeren Sinne hinausgeht und die Erhaltung und Steigerung von Lebensqualität ebenso umfasst wie Aspekte von Sorge und Optimierung bzw. Enhancement (Branch 2015; Savulescu 2007).

a) Lebensqualität ist in diesem Zusammenhang zu verstehen sowohl im Sinne von objektiven Lebensbedingungen und verfügbaren Optionen der Lebensgestaltung als auch im Sinne von persönlichen Werten, Standards, Anliegen (Group 1995): hier sind Aspekte der Selbstgestaltung und Weltgestaltung angesprochen.

b) Sorge im Sinne von Empathie, Würde und (Be-)Achtung von Vulnerabilität wie auch im Sinne einer Orientierung an individuellem emotionalen Wohlbefinden: hier sind Aspekte der Achtung vor dem Menschen in seiner Verletzlichkeit angesprochen (Rentsch 2019), wobei ich den Aspekt der Achtung 
noch um jenen der Demut vor dem Leben erweitert sehen möchte; in letztgenanntem Aspekt kommt auch die Ehrfurcht vor dem Leben (Schweitzer 1991) zum Ausdruck. Zudem sehe ich hier Anknüpfungspunkte zu Arbeiten von Immanuel Lévinas (1991), der als zentrale Form der Empathie die Fähigkeit und Bereitschaft des Menschen umschreibt, sich vom Antlitz des Anderen berühren zu lassen. Derartige sittlich-normative (und nicht nur fachliche) Qualitäten dürfen nicht aus dem Auge verloren werden, wenn es um die Integration von Robotertechnologie in diagnostische, therapeutische, vor allem in rehabilitative und pflegerische Akte geht.

c) Optimierung bzw. Enhancement im Sinne der Steigerung körperlicher und kognitiver Kapazität jenseits von spezifischen Therapiezielen. Auf diesen wichtigen Punkt, der auch Fragen der Gesundheitsförderung und Prävention berührt, werde ich im nachfolgenden Abschnitt zu sprechen kommen.

Non-maleficence: Das Prinzip des Nichtschadens verweist auf die Verpflichtung, die mit dem Einsatz von (nicht nur digitaler) Technologie verbundenen Risiken zu identifizieren, zu minimieren und im Kontext von Kosten-Nutzen-Erwägungen zu berücksichtigen. Zudem wird hier die Erhöhung von Sicherheit als eine grundlegende Zielsetzung des Einsatzes assistierender Technologien genannt; man denke nur an die frühzeitige Detektion körperlicher und kognitiver Risikofaktoren für einen Sturz. Zu berücksichtigen sind hier zudem die nicht intendierten Technikfolgen, die im Kontext von Evaluationsstudien (nicht nur im Prozess der Technikentwicklung, sondern auch nach der Implementierung von Technik) ermittelt werden können. Dabei stellt sich auch die Frage nach der Verantwortung in jenen Fällen, in denen ein ganzes Netzwerk von Verantwortungsträgern besteht, was zum Beispiel in stationären Einrichtungen der Fall ist. Wie wird die Verantwortung geteilt, wie wird diese kodifiziert und dokumentiert? Mir erscheint dieser Aspekt vor allem im Kontext der medizinischen, rehabilitativen und pflegerischen Versorgung von Menschen wichtig, bei denen eine deutlich erhöhte körperliche, vor allem eine deutlich erhöhte kognitive Vulnerabilität erkennbar ist.

Interdependence: Das Prinzip der Interdependenz verweist auf die grundlegende Bezogenheit des Menschen (auf Andere und Anderes) und die Zielsetzung intelligenter assistierender Technologien, Beziehungen zur sozialen und physikalischen (inklusive digitalen) Umwelt zu fördern oder zu ermöglichen: ein Beispiel für das Zusammenwirken von menschlicher und künstlicher Intelligenz. Zentrale Aspekte sind hier soziale Teilhabe, Einsamkeit und Verlust persönlich bedeutsamer Kontakte sowie Möglichkeiten, Teilhabe zu erhalten, Einsamkeit zu überwinden und den Verlust persönlich bedeutsamer Kontakte zu kompensieren. 
Justice: Das Prinzip der Gerechtigkeit postuliert eine faire Verteilung von Nutzen, Risiken und Kosten der Technologien; in meiner Diktion: die Verringerung, wenn nicht sogar Überwindung von digitaler Spaltung. Mit Blick auf intelligente assistierende Technologien sind insbesondere folgende Aspekte angesprochen: Gleichheit, fairer Zugang und Offenheit. Unter dem Aspekt der Gleichheit stellt sich die Frage, inwiefern Menschen in vergleichbaren Situationen bzw. mit vergleichbaren Bedarfen gleiche Voraussetzungen für die Nutzung der Technik haben bzw. in gleicher Weise von der Technik profitieren können. Die Forderung nach einem fairen Zugang bezieht sich sowohl auf die physikalische Verfügbarkeit von Technologie (z. B. in städtischen versus ländlichen Regionen) als auch auf gegebene Möglichkeiten zur Aneignung von Technologie (z. B. für verschiedene soziale Statusgruppen) und damit verbunden auf die Entwicklung erschwinglicher Technik unter der Zielsetzung der Vermeidung einer digitalen Spaltung der Gesellschaft, die bestehende Ungleichheiten weiter akzentuiert. Offenheit bezieht sich auf die (unter hinreichender Wahrung von geistigem Eigentum, Urheber- und Nutzungsrechten) möglichst freie Verfügbarkeit von Software-Codes, Lizenzen und Hardware-Komponenten.

Explicability/Transparency: Erklärbarkeit und Transparenz bilden eine grundlegende Voraussetzung für die Gewinnung und Aufrechterhaltung des Vertrauens potenzieller Nutzer in die Entwicklung digitaler Technologien. Dabei ist zu differenzieren zwischen Erklärbarkeit und Transparenz von Technologien - Systeme müssen prüffähig, nachvollziehbar und für Menschen mit unterschiedlicher Qualifikation und Nutzererfahrung verständlich sein - und Erklärbarkeit und Transparenz von Geschäftsmodellen - die Anwenderinnen und Anwender müssen hinreichend über die Intention der Entwicklung und Implementierung von Technologie informiert werden. Des Weiteren bezieht sich das Prinzip der Erklärbarkeit und Transparenz a) auf die Forderung nach einer informierten Zustimmung der von Technikanwendungen Betroffenen, b) auf die Notwendigkeit, Zuständigkeiten und Verantwortung für unerwünschte Technikfolgen zu klären, sowie c) auf den Nachweis des Nutzens der Entwicklung und Implementierung von Technologien.

\section{Ethische Dilemmata der Nutzung digitaler Technologie}

Aus der Nutzung digitaler Technologie ergeben sich sowohl spezifische Chancen als auch spezifische Risiken für Versorgungsqualität; Möglichkeiten des Einsatzes digitaler Technologie verweisen auf ethische Dilemmata, die im Zuge der 
Entwicklung, Nutzung und Finanzierung dieser Technologien bedacht werden müssen und gerade (aber nicht nur) dann, wenn jene, die von der jeweiligen Technologie profitieren sollen, in deren Nutzung nicht explizit und hinreichend informiert einwilligen können.

Im Kontext von digitaler Technologie in Diagnostik, Therapie, Rehabilitation und Pflege ergeben sich insbesondere Dilemmata aus Konflikten zwischen der Achtung der Autonomie und der Orientierung am Wohlergehen des Individuums. Der Einsatz zunehmend autonom agierender Technik ist mit der Gefahr verbunden, die Autonomie des Menschen zu missachten. Das Wohlergehen des Menschen erfordert die Berücksichtigung von Personalität, seine Einschätzung und Einfühlung, die (wenn überhaupt) schwer auf der Grundlage objektiv messbarer Kriterien zu operationalisieren ist. Ein besonderes Problem ist darin zu sehen, dass Eingriffe in die Autonomie des Menschen in vielen Fällen nicht durch eine informierte Einwilligung legitimiert werden, und Einschätzungen des Wohlergehens häufig nicht auf Willensäußerungen oder kommunizierte Präferenzen gegründet werden können. Demenziell erkrankte Menschen sind hier als eine besonders vulnerable Gruppe anzusehen (ausführlich in Kruse 2019; Lauter 2010).

\subsection{Ebene des Individuums}

Auf individueller Ebene ergeben sich Möglichkeiten der Förderung von Autonomie und Wohlergehen etwa durch die Unterstützung alltäglicher Routinen, kognitive Assistenzfunktionen (Information, Aktivierung), physische Dienstleistungen (Ernährung, Mobilität) oder die Erhöhung von Sicherheit (Monitoring). Dabei ergibt sich die Notwendigkeit, Schutz/Sicherheit und Selbstbestimmung gegeneinander abzuwägen und der Personalität der Pflegebedürftigen gerecht zu werden, insbesondere auch festzulegen, wann spezifische Voraussetzungen spezifische (automatisierte) Reaktionen notwendig machen. Nicht zu vernachlässigen sind dabei ethisch legitime Ansprüche von weiteren Akteuren im Versorgungsgeschehen (z. B. Angehörige, Professionelle), die auch unter ethischen Gesichtspunkten eine multiperspektivische Betrachtung erforderlich machen. Hier möchte ich den von Loewy und Springer-Loewy (2000) verwendeten Begriff der Orchestrierung aller Maßnahmen nennen, der zum einen die Kooperation zwischen den Berufsgruppen (darüber mit den Angehörigen) sehr gut veranschaulicht, der zum anderen deutlich macht, wie vielfältig die Bedürfnisse des schwerkranken oder sterbenden Menschen sind: 
auch daraus ergibt sich die enge Kooperation zwischen allen Akteuren im Versorgungsgeschehen und in welchem Maße diese ineinandergreifen (Loewy und Springer-Loewy 2000).

Während hinsichtlich der Forderung, dass im Kontext von Therapie, Rehabilitation und Pflege körperliche Schäden zu vermeiden sind, Konsens besteht, kann dies für die unter der entsprechenden Zielsetzung gerechtfertigten Mittel nicht vorausgesetzt werden. Hier muss zwischen dem Recht auf körperliche Unversehrtheit und weiteren Rechten, insbesondere der persönlichen Freiheit, dem Recht auf Schutz vor psychologischer Gewalt (u. a. auch Beeinträchtigungen des Sinn- und Kontrollerlebens) und dem Recht auf Privatheit abgewogen werden. Die Angemessenheit von Maßnahmen zur Gewährleistung von Sicherheit sollte wesentlich von der (psychischen und kognitiven) Gesundheit der auf Hilfe oder Pflege angewiesenen Person abhängig gemacht werden. Sharkey und Sharkey (2011) verdeutlichen dies etwa am Beispiel des Öffnens einer Schublade, die scharfe Messer enthält, und der damit verbundenen Gefahr der Selbstschädigung. Hier dürfte zunächst Einigkeit bestehen, dass Pflegefachkräfte in dieser Situation eine ältere Person nicht in ihrer Handlungsfreiheit beeinträchtigen sollten. Sofern es sich dagegen um einen älteren Menschen handelt, bei dem die Diagnose einer Suizidgefährdung vorliegt, dürfte das Unterbinden der Handlung eher angemessen, wenn nicht geboten sein. Während eine demenzkranke Person, die noch in der Lage ist, selbstständig im Privathaushalt zu leben, (eine nicht zu weit fortgeschrittene Erkrankung vorausgesetzt) nicht auf eine mögliche Gefahr aufmerksam zu machen oder gar in ihrer (vermeintlichen) Handlungsabsicht präventiv einzuschränken, wäre im Falle eines Menschen mit weiter fortgeschrittener Demenz eine entsprechende Intervention geboten. In ähnlicher Weise kann ein permanentes Monitoring u. U. im Kontext einer Intensivpflege als angemessen erachtet werden, während es im Kontext einer Teilzeitpflege oder der Verrichtung von Pflegetätigkeiten im Privathaushalt der Person als schwere Verletzung der Privatheit gelten kann, wenn die Person etwa beim Duschen oder beim Toilettengang beobachtet wird. Spezifische Probleme im Einsatz autonomer digitaler Technologie ergeben sich hier nicht allein aus der Notwendigkeit, eindeutig festzulegen, welche Reaktionen in welcher Situation notwendig und angemessen sind, und in diesem Zusammenhang auch die dem beobachteten Verhalten zugrunde liegenden Intentionen zuverlässig abzuschätzen. Unstrittig ist, dass Pflegefachkräfte hierzu gegenwärtig prinzipiell besser in der Lage sind als technische Systeme. Darüber hinaus ergibt sich das Problem, dass weder vorausgesetzt werden kann, dass sich die beobachteten Personen zum Zeitpunkt ihrer Handlungen darüber im Klaren sind, dass digitale Aufzeichnungen angefertigt werden, noch dass sie diesen in jedem Falle informiert zustimmen 
würden, zumal durch die Speicherung und Übertragung von Aufnahmen der Kreis potenzieller (mehr oder weniger befugter) Betrachter möglicherweise nicht mehr überschaubar ist (Deutscher Ethikrat 2018). Zu dem hier angesprochenen Risiko einer nicht gerechtfertigten Verletzung von Privatheit tritt die Gefahr einer Verletzung der Autonomie der Person hinzu. In diesem Zusammenhang verweist Sharkey (2014) auf ökonomische Erwägungen, die eine Ausweitung von Monitoring-Systemen hin zu weitgehend autonomen Überwachungssystemen wahrscheinlich machen. Da reines Monitoring, auf dessen Grundlage Pflegefachkräfte über die Notwendigkeit spezifischer Interventionen entscheiden können, mit einem vergleichsweise hohen Aufwand verbunden ist, liegt es nahe, Monitoring mit Robotik zu verbinden, die vor potenziell gefährlichen Aktivitäten warnen und diese ggf. auch aktiv unterbinden kann (etwa in dem Sinne, dass innerhalb eines Privathaushalts auf der Grundlage von Kartenmaterial Zonen ausgewiesen werden, die nicht betreten werden dürfen). Unabhängig davon, dass eine Einschränkung der persönlichen Freiheit im Interesse der Betroffenen im Einzelfall gerechtfertigt sein mag, besteht die Gefahr, dass autonome Systeme aus guten Gründen bestehenden rechtlichen Regelungen zuwider handeln könnten.

Bei der Begleitung sterbender Menschen können Monitoring-Systeme bis zum Lebensende (bis zur Feststellung des Todes) eingesetzt werden, wobei mit der Veränderung überwachter Vitalparameter prinzipiell autonom Interventionsmaßnahmen eingeleitet werden könnten. Gerade im Zusammenhang mit der Begleitung am Lebensende kann argumentiert werden, dass Kontakte zu anderen Menschen nicht durch Maschinen ersetzt und geregelt werden sollten. Des Weiteren wird gerade im Kontext der Sterbebegleitung deutlich, dass der Einsatz von Technik auch abgelehnt werden können sollte (dies auch im Sinne eines zur angemessenen Zeit Sterben-Dürfens).

\subsection{Ebene zwischenmenschlicher Beziehungen}

Auf der zwischenmenschlichen Ebene haben digitale Technologien das Potenzial, Möglichkeiten der Beziehungsgestaltung zu stärken, indem sie etwa durch die Übernahme von zeitaufwendigen oder belastenden Routinetätigkeiten (Hol- und Bringdienste, Hebetätigkeiten) Freiräume für Pflegefachkräfte ermöglichen. Gleichzeitig besteht die Gefahr, dass der Einsatz von digitaler Technologie eine Reduzierung sozialer Kontakte zur Folge hat, entstehende Freiräume mithin die Einsparung von Personal zur Folge haben. In diesem Zusammenhang kann darauf hingewiesen werden, dass ältere pflegebedürftige Menschen - in Privathaushalten wie in stationären Einrichtungen - ein erhöhtes Risiko für Einsamkeit 
und Isolation aufweisen und gerade hierin auch ein Grund dafür zu sehen ist, dass sie den Einsatz von Pflegerobotern in vielen Fällen ablehnen. Besuche von Pflegekräften sind für die Betroffenen möglicherweise so bedeutsam, dass auch in ansonsten schambesetzten Bereichen wie Körperhygiene Kontakt zu anderen Menschen als positiv erfahren und gesucht wird (Parks 2010; Sparrow und Sparrow 2006). Insbesondere mit Blick auf die Pflege bei fortgeschrittener Demenz könnte der Einsatz von digitaler Technologie im ungünstigen Falle Isolationstendenzen verstärken. Des Weiteren könnte ein Gewinn an Sicherheit zu einem geringeren Verantwortungsgefühl von Angehörigen beitragen. Empirische Studien machen deutlich, dass die Interaktion mit persönlichen Robotern wie der Pflegerobbe PARO positive Auswirkungen auf Einsamkeitsgefühle und Kommunikationsverhalten haben kann. Vor dem Hintergrund nachgewiesener Zusammenhänge zwischen sozialen Kontakten und kognitiver Leistungsfähigkeit sowie Einsamkeit und Demenz wie allgemein vor dem Hintergrund eines Rechts auf soziale Teilhabe ist festzustellen, dass emotionale Roboter soziale Kontakte allenfalls ergänzen, keinesfalls aber ersetzen können. In diesem Zusammenhang ist zu bedenken, dass digitale Technologien, die auf emotionale Befindlichkeiten und Kontaktbedürfnisse von Menschen reagieren, Bedürfnissen nach Bezogenheit schon deshalb nicht wirklich gerecht werden können, als sie empathische Reaktionen lediglich vortäuschen und - insofern sie auf Programmroutinen beruhen - der Individualität der Betroffenen gerade nicht gerecht werden. Das Bedürfnis nach Bezogenheit bezieht sich primär auf andere Menschen, darüber hinaus - biografisch bedingt - auf die erlebte Nähe zu Tieren (Preuß und Legal 2017) oder vertraute Gegenstände und Orte als Teil gewachsener Identität, nicht auf bis dato unvertrauten technischen Systemen und Robotern. Nicht übersehen werden sollte hier eine weitere, aus ethischer Perspektive weit positiver zu bewertende Option der Nutzung autonomer Systeme: deren Nutzung zur Anbahnung, Aufrechterhaltung und Anreicherung der zwischenmenschlichen Interaktion. PARO kann sich im konkreten Fall durchaus als ein probates Mittel für das Pflegepersonal erweisen, einen neuen Weg in der Kommunikation mit Menschen mit Demenz zu verfolgen. Dies verdeutlicht, dass die Gegenüberstellung von vollautomatisierter Betreuung und Versorgung und menschlicher Nähe dann in die Irre führt, wenn sie nicht im Sinne der Möglichkeit einer triadischen Kooperation ergänzt wird.

Die Auswirkungen assistierender Technologien auf zwischenmenschliche Kontakte sind gegenwärtig allerdings nicht primär darin zu sehen, dass sie Pflegefachkräfte ersetzen und so zur Isolation von Pflegebedürftigen beitragen. Entsprechende Szenarien orientieren sich weit stärker an möglichen Entwicklungen 
von Technik als an deren aktuell bestehenden Möglichkeiten und verfügbaren Anwendungen. Nicht übersehen werden sollte, dass durch Technologie auch spezifische Hilfen bereitgestellt werden, die Selbstständigkeit erhöhen und einen von Pflegefachkräften unabhängigeren Lebensstil ermöglichen können: ein Potenzial der Robotertechnologie.

\subsection{Ebene der Gesellschaft}

Auf gesellschaftlicher Ebene ergibt sich die Notwendigkeit, neben der Bewertung aktuell verfügbarer oder im Sinne von Visionen antizipierter Technikprodukte den Prozess verantwortlicher Forschung und Entwicklung stärker in den Blick zu nehmen. Weiterhin stellt sich die Frage, wie mit digitaler Technologie verbundene Chancen und Lasten (Finanzierung) gerecht verteilt werden können. In diesem Zusammenhang wäre zwischen gesellschaftlicher Verteilungsgerechtigkeit und individueller Bedarfsgerechtigkeit zu differenzieren. Letztere kann vor dem Hintergrund des bereits erwähnten Befähigungsansatzes im Sinne von Befähigungsgerechtigkeit diskutiert werden. In diesem Zusammenhang wurde die Befürchtung geäußert, dass unter der Voraussetzung der Verfügbarkeit effektiver Technologie technologische Leistungen gegenüber personalen Leistungen aus ökonomischen Erwägungen vorrangig gewährt werden könnten, was im Einzelfall zur Folge haben kann, dass befähigende Hilfe zur Selbsthilfe Aspekte der Bedarfsgerechtigkeit vernachlässigt (Remmers 2018, 2019).

\subsection{Folgerungen}

Es liegt in der Natur ethischer Dilemmata, dass verschiedene Handlungen und Unterlassungen, die in einer spezifischen Entscheidungssituation geboten erscheinen, nicht miteinander zu vereinbaren sind, spezifische Chancen zugunsten anderer aufgegeben, spezifische Risiken im Dienste der Vermeidung anderer in Kauf genommen werden müssen. Entsprechend lassen sich die beschriebenen, im Kontext ethischer Entscheidungen zu berücksichtigenden Prinzipien nicht verbindlich ordnen, z. B. in dem Sinne, dass Einschränkungen von Freiheit, Autonomie und Privatheit gerechtfertigt wären, wenn damit nachgewiesenermaßen Schaden vermieden oder zur Verwirklichung von Aspekten objektiver Lebensqualität beigetragen wird. Allgemeine ethische Prinzipien stellen sich gerade auch für die von Verletzungen (potenziell) Betroffenen kontext- wie personenspezifisch zum Teil sehr unterschiedlich dar (Kruse 2017). Verletzungen von 
Privatheit in Form einer Speicherung und Weiterverarbeitung personenbezogener Daten im Kontext der Nutzung digitaler Dienstleistungs- und Unterhaltungsangebote dürften etwa von den meisten Menschen anders bewertet werden als Verletzungen im Kontext gesundheitlicher Versorgung oder im Kontext der individuellen Absicherung von Risiken. Des Weiteren dürfte das, was manchen als in Art und Ausmaß unter keinen Umständen hinnehmbare Verletzung persönlicher Rechte erscheint, von anderen vor dem Hintergrund sich daraus (mutmaßlich) ergebender Vorteile als unbedeutend erscheinen. Vor diesem Hintergrund erscheint es weder möglich noch wünschenswert, Dilemmata aus konkurrierenden ethischen Perspektiven inhaltlich (im Sinne einer jeweils verbindlich zu präferierenden Alternative) zu lösen. Die beschriebenen Dilemmata verweisen vielmehr auf die Frage, wie Entscheidungsprozesse - im Allgemeinen wie im konkreten Fall - zu gestalten sind. Hier dürfte Einigkeit bestehen, dass Menschen grundsätzlich das Recht zukommt, Technologie nicht zu nutzen bzw. der Anwendung von Technik im Einzelfall zu widersprechen - dies unabhängig von einem von anderen erwarteten Nutzen: Menschen dürfen etwa Behandlungen ablehnen, sie haben im Übrigen auch das Recht, sich selbst zu schaden, Präventions- und Kompensationsmöglichkeiten nicht zu nutzen, vermeidbare Risiken einzugehen (Deutscher Ethikrat 2018).

Im Kontext der Entwicklung, Implementierung und Nutzung digitaler Technologie ergeben sich darüber hinaus aber weitere Probleme. Zunächst benötigen Menschen Informationen, um sich zwischen Alternativen entscheiden zu können, wobei sich die individuelle Einschätzung von Chancen und Risiken über die Zeit verändern können - mit zunehmenden Erfahrungen im Umgang mit Technik (eigenem Handeln wie Handeln und Reaktionen anderer) wie allgemein mit Veränderungen der Lebenssituation. Sodann ist zu berücksichtigen, dass im Falle gravierender Einschränkungen der Urteilsfähigkeit auch stellvertretend für die Betroffenen (vor dem Hintergrund deren mutmaßlich leitender Bedürfnisse und Präferenzen) entschieden werden muss (auch der Verzicht auf den Einsatz von Technik ist in diesem Zusammenhang begründungsbedürftig). Des Weiteren ist die Verfügbarkeit von Alternativen bereits das Ergebnis (auch) ethischer Entscheidungen, insofern technische Artefakte ausgehend von gesellschaftlichen Problemdefinitionen und Lösungsvisionen entwickelt werden. Daraus ergibt sich die Forderung, im Prozess der (Weiter-)Entwicklung von digitaler Technologie Chancen und Risiken aus zum Teil sehr unterschiedlichen Perspektiven (nicht nur jener der potenziellen Nutzer, sondern auch anderer in die Entwicklung, Nutzung, Verbreitung und Vermarktung involvierter Personen) sensibel zu reflektieren. In diesem Zusammenhang hat sich im Kontext der öffentlichen Förderung von 
Technologieentwicklung die Forderung nach einer ethisch informierten Begleitforschung durchgesetzt (Kommission 2020). Derartigen Bemühungen vorgeordnet ist allerdings die Notwendigkeit eines gesellschaftlichen Diskurses, der sich nicht wie ethisch informierte Begleitforschung primär auf das wie, sondern grundlegender auf das $o b$ konzentriert - im Zusammenhang mit Enhancement als zentrales Anwendungsfeld digitaler Technologie wurde auf diesen Punkt ausführlicher eingegangen. Des Weiteren ergeben sich aus der prinzipiellen Verfügbarkeit von Technologie Gerechtigkeitsfragen, die gleichfalls nur auf einer gesellschaftlichen Ebene (ggf. auch durch die Etablierung von justiziablen Rechten und Pflichten) beantwortet werden können. Klar dürfte sein, dass die Vorstellung, technologische Innovationen bzw. spezifische technische Artefakte könnten allen Menschen in identischer Weise zugängig sein, nicht nur naiv, sondern auch nicht wünschenswert ist. Vielmehr geht es darum, zu klären, welche Möglichkeiten von Technik vor dem Hintergrund spezifischer Bedarfe jeweils in welchem Umfang zur Verfügung zu stellen und ggf. auch solidarisch zu unterstützen oder zu finanzieren sind (Kommission 2020).

\section{Abschluss}

Die Zielsetzung des Beitrags bestand darin, Selbstgestaltung und Weltgestaltung als grundlegende Bedürfnisse und Orientierungen der Person - in ihrer Bedeutung für die Anwendung von Künstlicher Intelligenz, hier vor allem der Robotertechnologie aufzuzeigen. Dabei ließ sich der Beitrag von der Annahme leiten, dass Künstliche Intelligenz, dass Robotertechnologie in keinem Gegensatz zu personalen Entscheidungs- und Handlungsprozessen stehen, sondern diese in fruchtbarer, die Selbst- und Weltgestaltung fördernder Art und Weise bereichern können. Diese Bereicherung ergibt sich in dem Maße, in dem diese Technologie harmonisch, man könnte vielleicht auch sagen: organisch in die Lebenswelt und das Handlungsspektrum der Person integriert wird, was auch bedeutet, dass die Person durch diese Technologie befähigt wird, bestimmte Handlungen auszuführen, die ihr sonst - so zum Beispiel im Falle der Einbußen in den für die Lebensgestaltung zentralen Funktionen und Fertigkeiten - nicht möglich wären. Der Erfolg in der Umsetzung dieser Technologien ist auch davon abhängig, inwieweit die Person befähigt wird, die Technologie in ihrem potenziellen Einfluss auf die eigene Lebenswelt, auf das eigene Handeln zu verstehen und zu nutzen: damit ist ein bedeutender Bildungsauftrag angesprochen.

Dass dem Autor die Aufgabe übertragen war, bei der Vorbereitung auf den - diesem Beitrag zu-zugrunde liegenden - Vortrag die Musik von Johann 
Sebastian Bach, hier Präludium und Fuge in cis-Moll aus dem Wohltemperierten Klavier I, mit ethischen Reflexionen über die Anwendung von Robotertechnologie zusammenzuschauen, war alles andere als der Anstoß eines Versuchs, inkommensurabel erscheinende Inhalte künstlich zusammenzuführen. Vielmehr lag darin die Aufforderung, Überlegungen darüber anzustellen, wie die Musik von Johann Sebastian Bach auch für ethische Reflexionen fruchtbar gemacht und in die hier geführte Diskussion eingebracht werden kann. Die Annahme, die hier aufgestellt und begründet wurde, lautete: die Musik von Johann Sebastian Bach - und hier vor allem die Kompositionsform der Fuge - ist in besonderer Weise geeignet, Prozesse der Selbstreflexion anzustoßen, die Introversion mit Introspektion zu fördern. Die Introversion mit Introspektion - von mir als eine grundlegende Orientierung im hohen Alter angesehen (Kruse 2017) - ist bedeutsam, wenn es darum geht, die Potenziale (seelisch-geistige Reifung) wie auch die Grenzen (körperliche, kognitive, emotionale Verletzlichkeit) in der gegenwärtigen Situation zu erkennen und auf diese verantwortungsvoll $\mathrm{zu}$ antworten, wobei die Verantwortung nicht nur sich selbst gegenüber, sondern auch dem sozialen Nahumfeld gegenüber besteht. Weiterhin sind die in der persönlichen Zukunft liegenden Potenziale und Grenzen zu bedenken (Antizipation). In einem solchen Prozess der Selbstreflexion, der Introversion und Introspektion gewinnt auch die Frage an Bedeutung, inwieweit Technik in die eigene Lebenswelt und in das eigene Handlungsspektrum integriert werden kann und welche Entwicklungs-, Anpassungs- und Veränderungsprozesse der eigenen Person notwendig sind, um diese Integration zu ermöglichen.

\section{Literatur}

Arendt, H. (1960). Vita Activa oder vom tätigen Leben. Stuttgart: Kohlhammer.

Arendt, H. (1989). Gedanken zu Lessing. Von der Menschlichkeit in finsteren Zeiten. In H. Arendt (Hrsg.), Menschen in finsteren Zeiten (S. 3-36). München: Piper.

Arendt, H. (1993). Was ist Politik? München: Piper.

Baltes, M. M. (1996). The many faces of dependency in old age. New York: Cambridge University Press.

Blumenberg, H. (1986). Lebenszeit und Weltzeit. Frankfurt: Suhrkamp.

Branch, W., Jr. (2015). A piece of my mind. The ethics of patient care. JAMA, 313(14), 1421-1422.

Brandtstädter, J. (2007). Konzepte positiver Entwicklung. In J. Brandtstädter \& U. Lindenberger (Hrsg.), Entwicklungspsychologie der Lebensspanne (S. 681-723). Stuttgart: Kohlhammer.

Brandtstädter, J. (2014). Lebenszeit, Weisheit und Selbsttranszendenz Aufgang - Jahrbuch für Denken, Dichten, Musik (Bd. 11, S. 136-149). Stuttgart: Kohlhammer. 
Brothers, A., Gabrian, M., Wahl, H.-W., \& Diehl, M. (2016). Future time perspective and awareness of age-related change: Examining their role in predicting psychological wellbeing. Psychology and Aging, 31, 605-617.

Buonarroti, M. (2002). Zweiundvierzig Sonette (Übers. von R. M. Rilke). Frankfurt a. M: Insel.

Butler, R. N. (1963). The life review: An interpretation of reminiscence in the aged. Psychiatry, 26, 65-76.

Clegg, A., Young, J., Iliffe, S., Rikkert, M. O., \& Rockwood, K. (2013). Frailty in elderly people. Lancet, 381, 752-762.

Dentler, H. E. (2004). Johann Sebastian Bachs „Kunst der Fuge“ Ein pythagoreisches Werk und seine Verwirklichung. Mainz: Schott.

Deutscher Ethikrat. (2018). Big Data und Gesundheit - Datensouveränität als informationelle Freiheitsgestaltung. Stellungnahme. Berlin: Deutscher Ethikrat.

Deutschen Ethikrat. (2020). Robotik für gute Pflege. Stellungnahme. Berlin: Deutscher Ethikrat.

Eggebrecht, H. H. (1998). Bachs Kunst der Fuge. Erscheinung und Deutung (4. Aufl.). Wilhelmshaven: Florian Noetzel.

Erikson, E. H. (1998). The life cycle completed. Extended version with new chapters on the ninth stage by Joan M. Erikson. New York: Norton.

European Commission. (2018). High-Level Expert Group on Artifical Intelligence. Draft. Ethics Guidelines for Trustworthy AI. Brussels: European Commission.

Fiske, A., Henningsen, P., \& Buyx, A. (2019). Your robot therapist will see you now: Ethical implications of embodied artificial intelligence in psychiatry, psychology, and psychotherapy. Journal of Medical Internet Research, 21(5), e13216. https://doi. org/10.2196/13216

Fried, L. P., Tangen, C. M., Walston, J., Newman, A. B., Hirsch, C., Gottdiener, J., et al. (2001). Frailty in older adults: Evidence for a phenotype. Journal of Gerontology, 56A, $146-156$.

Kommission. (2020). Ältere Menschen und Digitalisierung. Achter Bericht zur Lage der älteren Generation in der Bundesrepublik Deutschland. Berlin: Bundesministerium für Familie, Senioren, Frauen und Jugend.

Kruse, A. (2005a). Selbstständigkeit, Selbstverantwortung, bewusst angenommene Abhängigkeit und Mitverantwortung als Kategorien einer Ethik des Alters. Zeitschrift für Gerontologie und Geriatrie, 38, 273-287.

Kruse, A. (2005b). Biografische Aspekte des Alter(n)s: Lebensgeschichte und Diachronizität. In U. Staudinger \& S.-H. Filipp (Hrsg.), Enzyklopädie der Psychologie, Entwicklungspsychologie des mittleren und höheren Erwachsenenalters (S. 1-38). Göttingen: Hogrefe.

Kruse, A. (2014). Die Grenzgänge des Johann Sebastian Bach - Psychologische Einblicke (2. Aufl.). Heidelberg: Springer Spektrum.

Kruse, A. (2015). Resilienz bis ins hohe Alter - was wir von Johann Sebastian Bach lernen können. Heidelberg: Springer.

Kruse, A. (2017). Lebensphase hohes Alter - Verletzlichkeit und Reife. Heidelberg: Springer. 
Kruse, A. (2019). Demenz als Herausforderung an gelingendes Sterben. In O. MitscherlichSchönherr (Hrsg.), Gelingendes Sterben Zeitgenössische. Theorien im interdisziplinären Dialog (S. 177-204). Berlin: deGruyter.

Kruse, A., \& Schmitt, E. (2015). Shared responsibility and civic engagement in very old age. Research in Human Development, 12, 133-148.

Kruse, A., \& Schmitt, E. (2016). Sorge um und für andere als zentrales Lebensthema im sehr hohen Alter. In J. Stauder, I. Rapp, \& J. Eckhard (Hrsg.), Soziale Bedingungen privater Lebensführung (S. 325-352). Heidelberg: Springer.

Kruse, A., \& Schmitt, E. (2018). Spirituality and transcendence. In R. FernándezBallesteros, A. Benetos, \& J.-M. Robine (Hrsg.), Cambridge Handbook of Successful Aging (S. 426-454). Cambridge: Cambridge University Press.

Lauter, H. (2010). Demenzkrankheiten und menschliche Würde. In A. Kruse (Hrsg.), Lebensqualität bei Demenz? Zum gesellschaftlichen und individuellen Umgang mit einer Grenzsituation im Alter (S. 27-42). Heidelberg: Akademische Verlagsgesellschaft.

Lévinas, I. (1991). Entre nous. Essais sur le penser-à-l'autre. Paris: Grasset \& Fasquelle. (Deutsch: [1995]. Zwischen uns. Versuche über das Denken an den Anderen. München: Hanser.)

Loewy, E., \& Springer-Loewy, R. (2000). The ethics of terminal care. Orchestrating the end of life. New York: Kluwer Academics.

Mirandola, P.G., d. (1496/1990). De hominis dignitate (Deutsch: Über die Würde des Menschen). Hamburg: Meiner.

Nietzsche, F. (1878/1998). Menschliches, Allzumenschliches. Berlin: De Gruyter.

Nussbaum, M. C. (2011). Creating capabilities: The human development approach. Cambridge: Belknap Press of Harvard University Press.

Parks, J. (2010). Lifting the burden of women's care work. Should robots replace the "human touch"? Hypatia, 25(1), 100-120.

Peck, R. (1968). Psychologische Entwicklung in der zweiten Lebenshälfte. In H. Thomae \& U. Lehr (Hrsg.), Altern - Probleme und Tatsachen (S. 376-384). Wiesbaden: Wissenschaftliche Buchgesellschaft.

Preuß, D., \& Legal, F. (2017). Living with the animals: Animal or robotic companions for the elderly in smart homes? Journal of Medical Ethics, 43, 407-410.

Remmers, H. (2018). Pflegeroboter: Analyse und Bewertung aus Sicht pflegerischen Handelns und ethischer Anforderungen. In O. Bendel (Hrsg.), Pflegeroboter (S. 161180). Wiesbaden: Springer.

Remmers, H. (2019). Pflege und Technik. Stand der Diskussion und zentrale ethische Fragen. GKV-Spitzenverband. (Hrsg.), Digitalisierung und Pflegebedürftigkeit - Nutzen und Potenziale von Assistenztechnologien (S. 225). Hürth: Haarfeld.

Rentsch, T. (2019). Das Gelingen des Lebens im hohen Alter - Sieben Thesen. In O. Mitscherlich-Schönherr (Hrsg.), Gelingendes Sterben. Zeitgenössische Theorien im interdisziplinären Dialog (S. 73-84). Berlin: deGruyter.

Ritschl, D. (2004). Metaphorik der Anthropologie der Zeit. In D. Ritschl (Hrsg.), Zur Theorie und Ethik der Medizin. Philosophische und theologische Anmerkungen (S. 53-59). Neukirchen-Vluyn: Neukirchener.

Savulescu, J. (2007). In defence of procreative beneficence. Journal of Medical Ethics, 33(5), 284-288. 
Schweitzer, A. (1991). Die Ehrfurcht vor dem Leben - Grundtexte aus fünf Jahrzehnten (6. Aufl.). München: Beck.

Sharkey, A. (2014). Robots and human dignity: A consideration of the effects of robot care on the dignity of older people. Ethics and Information Technology, 16, 63-75.

Sharkey, N., \& Sharkey, A. (2011). The Rights and Wrongs of Robot Care. In C. Allen, W. Wallach, J. J. Hughes, S. Bringsjord, J. Taylor, N., Sharkey, \& R. O'Meara (Hrsg.), Robot ethics: The ethical and social implications of robotics (S. 267-282). Cambridge: MIT Press.

Sharkey, A., \& Sharkey, N. (2012). Granny and the robots: Ethical issues in robot care for the elderly. Ethics and Information Technology, 14(1), 27-40.

Staudinger, U. (1996). Psychologische Produktivität und Selbstentfaltung im Alter. In M. M. Baltes \& L. Montada (Hrsg.), Produktives Leben im Alter (S. 344-373). Frankfurt a. M.: Campus.

Staudinger, U. M. (2015). Images of aging: Outside and inside perspectives. Annual Review of Gerontology and Geriatrics, 35, 187-209.

Sparrow, R., \& Sparrow, L. (2006). In the hands of machines? The future of aged care. Mind and Machine, 16, 141-161.

Thomae, H. (1968). Das Individuum und seine Welt. Göttingen: Hogrefe.

Wolff, Ch. (2009). Johann Sebastian Bach (3. Aufl.). Frankfurt a. M.: Fischer.

Open Access Dieses Kapitel wird unter der Creative Commons Namensnennung 4.0 International Lizenz (http://creativecommons.org/licenses/by/4.0/deed.de) veröffentlicht, welche die Nutzung, Vervielfältigung, Bearbeitung, Verbreitung und Wiedergabe in jeglichem Medium und Format erlaubt, sofern Sie den/die ursprünglichen Autor(en) und die Quelle ordnungsgemäß nennen, einen Link zur Creative Commons Lizenz beifügen und angeben, ob Änderungen vorgenommen wurden.

Die in diesem Kapitel enthaltenen Bilder und sonstiges Drittmaterial unterliegen ebenfalls der genannten Creative Commons Lizenz, sofern sich aus der Abbildungslegende nichts anderes ergibt. Sofern das betreffende Material nicht unter der genannten Creative Commons Lizenz steht und die betreffende Handlung nicht nach gesetzlichen Vorschriften erlaubt ist, ist für die oben aufgeführten Weiterverwendungen des Materials die Einwilligung des jeweiligen Rechteinhabers einzuholen.

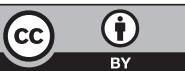

